


\title{
Las ciencias sociales y la comunicación para la paz en contextos en que persiste la violencia
}

\author{
Santiago Álvarez*
}

Recuerdo haberme encontrado por casualidad hace unos años, en una librería de Bogotá, con Jesús Antonio Bejarano, quien fue Comisionado para la Paz y murió luego trágicamente asesinado. En ese encuentro, en el que fuimos presentados por una amiga, él me dedicó un ejemplar de su libro Una agenda para la paz. En su obra, Bejarano trabajaba desde la teoría de la resolución de conflictos, buscando una salida política para la situación colombiana. Transcurría el gobierno de Samper; en ese entonces había un cierto optimismo acerca de la posibilidad de restablecer las conversaciones de paz con las Fuerzas Armadas Revolucionarias de Colombia (FARG). Bejarano consideraba que ese no era el momento para discutir interminablemente sobre la violencia, sino que era el momento para la acción política. Recuerdo que hizo una serie de críticas muy agudas, formuladas de

\footnotetext{
* Doctor en Antropología de la London School of Economics and Political. Actualmente forma parte del Centro de Antropología del Instituto de Desarrollo Económico y Social (IDES) y es profesor de la maestría en Antropología Social IDES de la Universidad San Martín. Correo electrónico: alvaresantiago@hotmail.com
} 
manera particularmente simpática, hacia quienes denominaba -y en la discusión, de alguna manera me hacía sentir incluido en esa denominación"violentólogos": personas que aparentemente desarrollan un alto saber especulativo sobre la violencia, pero que carecían de perspectivas prácticas para resolver conflictos violentos.

De esto quiero hablar: de las dificultades y desconexiones entre las investigaciones sobre la violencia y las actividades prácticas tendentes a resolver conflictos. Tenemos, por un lado, políticas que buscan -y a veces logranllegar a acuerdos que permitan resolver puntos de partida básicos para la convivencia. Tenemos, por otra parte, personas que intentan comunicar mensajes sobre la violencia, procurando modificar hábitos o crear alarma sobre tal o cual problemática; incluso contamos con investigadores que trabajan en una esfera más especulativa, sin casi relación con lo pragmático, para quienes la violencia parece ser importante en cuanto tema a pesquisar, más que en cuanto tema a develar o resolver.

Teniendo en cuenta estas dispersiones, me interesa no solo reflexionar sobre el pensar y el comprender la violencia, sino también sobre cómo crear canales que permitan utilizar ese conocimiento para transformar la realidad. Los investigadores sociales tenemos una aguda tendencia a hacer las cosas más complejas y, por tanto, más difíciles. ¿Sirve tener una perspectiva más compleja? Voy a tratar de dar algunos ejemplos de por qué sirve el análisis sobre la violencia -y, particularmente, de por qué sirve complejizarlo- en una estrategia comunicacional de cambio.

\section{La relación entre aplicación práctica y estudio especulativo}

Para desarrollar mi punto de vista, tal vez sea una buena idea empezar dando un breve ejemplo de otra realidad social y política, en este caso de Argentina. Realicé un estudio en mi país, a fines de los años noventa, sobre representaciones de la criminalidad en la justicia argentina ante lo que se 
denominaba la "nueva ola" de violencia criminal. Este estudio recababa información sobre lo que pensaban los actores de la justicia, incluyendo en ella no solo a jueces o fiscales, sino también a abogados de matrícula. En el trabajo emergía una constante referencia, por parte de los actores de la justicia, al hecho de que para los medios y para el ciudadano común cuando se hablaba de inseguridad o violencia, se hacía alusión fundamentalmente a la violencia denominada "de caño" (la violencia por robo a mano armada), a la violación y al homicidio (Álvarez, 2002). Esos delitos configuraban el centro de lo que los medios de comunicación denominaban "inseguridad".

En un trabajo publicado en la misma compilación en la que aparecieron los resultados parciales de mi investigación, Pablo Bonaldi estudiaba "Muertes violentas en la Argentina de 1980 a 1999”. Él entendía por muertes violentas "al conjunto de defunciones producidas por accidentes de tránsito, suicidios, homicidios u otro tipo de accidentes" (Bonaldi, 2002, p. 277). En 1999, en Argentina se habían producido 2431 suicidios, 1930 homicidios, 4651 muertes en accidentes de tránsito y 7000 en accidentes de otro tipo (Bonaldi, 2002). Podemos observar, entonces, cómo la inseguridad causada por accidentes, en particular por accidentes de tránsito, era casi absolutamente dejada de lado por los comunicadores sociales de los principales medios de comunicación, y no era tampoco percibida como uno de los problemas centrales de inseguridad por el ciudadano común, si bien los accidentes de tránsito eran causantes de casi tres veces más muertes que los homicidios dolosos.

Además, no había una política nacional tendente a dar respuesta al problema de la accidentalidad (la hubo más adelante, aunque siempre ocupando un espacio mediático menor). Como anécdota personal, habiendo regresado al país después de un largo periodo en el exterior, me costaba entender que personas que despotricaban constantemente sobre el aumento de la criminalidad dejasen que sus hijos menores de seis años viajasen en los asientos de los automóviles sin ponerles el cinturón de seguridad. Puede verse en este simple ejemplo cómo un estudio científico nos invita 
a replantear nuestras ideas sobre los problemas que urge resolver y, en el caso de una estrategia comunicacional, a corregir el objetivo hacia dónde debemos apuntar.

\section{Conexión entre distintos tipos de violencia, complejidad y cultura}

Como para discutir el fenómeno de la violencia desde las ciencias sociales es necesario precisar qué queremos decir con tal noción, adoptaré una mínima definición transcultural de violencia, a saber: "una resistida producción de mal físico" ("a contestable rendering of physicalhurt", Riches, 1988, p. 28). O, en el mismo sentido, la definición de Marvin (1988): "Toda acción humana que supone la deliberada inflicción de daño hacia otros” (p. 121). Haré uso de estas definiciones porque, por un lado, considero que son lo suficientemente amplias para incluir casos tales como insultos verbales y acosos en la esfera doméstica; por otro lado, porque estas concepciones imponen algunos límites sobre lo que debe ser considerado violento. Así, no haré uso del concepto "violencia" para describir toda acción social que implique contacto físico o comunicación verbal. Esta estrategia no abarca al concepto bourdiano de violencia simbólica, que describe formas de violencia no ejercidas directamente mediante la fuerza física, sino a través de la imposición, por parte de los sujetos dominantes a los sujetos dominados, de una cosmovisión de roles sociales específicos, de categorías cognitivas y de estructuras mentales.

Señalé anteriormente, de forma breve, un caso argentino con la intención de introducirnos en la discusión sobre la relación entre investigación social, utilidad política y comunicación social. Desarrollaré ahora más extensamente la realidad colombiana, tratando de dar una respuesta, en nombre de los "violentólogos", a Bejarano, por lo que me centraré en tomar mi trabajo de campo en Colombia como eje para desarrollar mis ideas. 
El pueblo de Nómeque -así denominaré a la comunidad en donde realicé mi estudio - se veía afectado por diferentes expresiones de violencia externa e interna. Varias familias campesinas estaban enfrentadas en venganzas de sangre, en las que ideales agresivos de masculinidad encontraban su trágica expresión. Por otra parte, una palpable violencia doméstica revelaba un conflicto por el control del hogar y probaba la existencia de tensiones expresadas en abusos físicos o verbales dirigidos contra las mujeres y los niños. Además de esto, diversos actores sociales -la guerrilla, los narcotraficantes, las fuerzas armadas- luchaban violentamente por el control de la región.

Durante mi trabajo de campo, varios miembros de la comunidad, situados por voluntad o por azar en medio de estos conflictos, fueron asesinados. Estas diferentes manifestaciones de violencia estaban conectadas, y sus actores a menudo cumplían en ellas múltiples roles sociales. En mi tesis he tratado de separar conscientemente estas situaciones por motivos analíticos, pero en la práctica se encontraban interrelacionadas y se retroalimentaban. Por ello, es importante explorar de qué manera los distintos tipos de violencia estaban interrelacionados y se influenciaban mutuamente.

\section{Patronazgo-madrazgo}

Siguiendo con mi idea inicial de hacer más complejo el discurso, quisiera hacer referencia al hecho de que en Nómeque era posible observar tensiones o valores antagónicos entre ideas de individualismo y solidaridad campesina, y entre jerarquía e igualitarismo. El análisis de los conflictos dentro de la familia y de sus valores y representaciones me ayudó a entender la violencia interna, lo que a su vez fue una clave para comprender la relación entre las fuerzas externas y la comunidad. En mi tesis doctoral procuré explicar cómo una representación de la familia devenía una representación política.

Como vemos, un análisis de una comunidad en toda su diversidad no se trata de una mirada direccionada solo hacia las relaciones políticas, ni 
de una mirada centrada únicamente en las relaciones familiares: la relación entre ellas nos zambulle en un intercambio intrincado y complejo. Encontré en la comunidad un conjunto de valores que consideré hegemónico y que expresaba ideas de superioridad masculina y jerarquía; sin embargo, descubrí también resistencia a este conjunto dominante, una resistencia basada en ideas de igualdad y solidaridad. En particular, procuré mostrar el modo en que ideas extremas de masculinidad están relacionadas con la política y el poder.

Utilicé el término "patronazgo" para referirme a un sistema de valores y prácticas que estaba relacionado con una forma particular de sociedad patriarcal y que articulaba la conexión entre lo político y las representaciones acerca de la masculinidad. El concepto de patronazgo deriva de la palabra española "patrón", que hace referencia al antiguo sistema de haciendas dominado por esa poderosa figura del patrón (Mintz y Wolf, 1957). Él, con dudosos derechos sobre la tierra, explotó la fuerza de trabajo campesina para desmontar amplias extensiones. Para hacerlo, se sirvió a la vez de la fuerza y la seducción, la violencia y la ambigüedad. El vínculo de los campesinos con sus patrones era de obligación, a causa del poder superior que ejercían estos últimos; por tanto, más allá de lo ritualizada que estuviese, la relación escondía siempre un velado conflicto.

Si analizamos la historia particular del Sumapaz, nos encontramos con que los campesinos resistieron al sistema de haciendas y se rebelaron contra sus patrones; así lograron finalmente ocupar y dividir la tierra de las grandes haciendas. El ocaso del perimido sistema (el tiempo me impide desarrollar este punto de un modo que no sea brutalmente sintético) no eliminó, sin embargo, las ideas y valores relacionados con él. En el momento en que realicé mi trabajo de campo pude ver esos mismos valores reflejados en el intento de los narcotraficantes de convertirse en los nuevos patrones, a través de la reconstrucción exitosa de las jerarquías existentes. En la interpretación que realicé de las fuentes culturales del surgimiento de los narcotraficantes como nueva clase consideraba que la recreación de estas relaciones 
jerárquicas entre patrones y clientes tenía raíces en valores campesinos. Analizaba, entonces, ideas y estereotipos acerca de los roles femeninos y masculinos en relación con sus prácticas concretas. Mi intención era mostrar cómo el sistema de valores del patronazgo influenciaba directamente la organización de la comunidad e implicaba la aceptación de un orden jerárquico. Estas ideas jerárquicas tenían raíces profundas en la historia de la región, particularmente en el orden hispánico colonial, en la figura del conquistador y, como ya señalé, en el antiguo régimen de haciendas.

Consideré que el concepto "patronazgo" definía una particular versión del sistema patriarcal y estaba relacionado con un tipo específico de organización familiar. Idealmente, este tipo estaba dominado por una figura masculina poderosa y central que ejercía control sobre mujeres (con la posibilidad de tener más de una compañera sexual permanente), sobre una numerosa prole (legítima e ilegítima) y, además, sobre hombres subordinados a ella. Este hombre era amo y señor de la propiedad familiar, especialmente la tierra, ejerciendo poder sobre un territorio específico (Reinhardt, 1988). No obstante, en Nómeque, los intentos de los hombres de actuar este rol y de imponer su poder eran solo parcialmente exitosos.

Algunos colegas me sugirieron el uso de los términos "macho" o "machismo" para referirme a este conjunto dominante de valores y prácticas (Lewis, 1970). Desde mi punto de vista, encontré que estos términos eran demasiado vagos y ambiguos para el propósito de mi estudio: ambos habían sido usados en exceso por autores envueltos de manera activa en debates políticamente correctos, para describir modelos extremos de estereotipos masculinos, particularmente en el Mediterráneo y en Latinoamérica. Como Stevens (1973) hizo notar hace más de veinte años, "machismo" es "un término familiar para especialistas en el área, [que] ha pasado al vocabulario del gran público, donde ha sufrido el mismo tipo de deformación semántica que el weberiano término 'carisma”' (p. 90; Melhus y Stolen, 2008). 
El uso de los conceptos "macho" o "machismo" implicaba poner el foco en el estudio de ideologías de género, lo cual oscurecía una comprensión más general del problema, compuesto por una suma de complejos elementos sociales y culturales. El concepto "machismo" aislaba elementos de sexo y género, mientras que yo estaba especialmente interesado en la articulación de estos elementos con lo político. Los términos "cacique" y "caciquismo" eran también limitados. Estos conceptos, al contrario que el de machismo, estaban limitados esta vez a lo político, particularmente a una expresión de representación política percibida como anómala, oscureciendo, en este caso, la importancia de los elementos sexuales y de género (Cruz Artacho, 1994; Pitt-Rivers, 1954).

Por otra parte, el concepto "honor" había sido utilizado para describir no solo un conjunto de valores, sino además para poner en relación esos valores "al nivel de la conducta" (Peristiany y Pitt Rivers, 1992), dando cuenta así de amplios aspectos de las sociedades mediterráneas. Había considerado la utilización del concepto "honor" en vez del concepto "patronazgo"; sin embargo, la idea de pureza femenina asociada al concepto de honor en las sociedades mediterráneas no me parecía central en la vida de la comunidad analizada, ni siquiera en los casos de vendettas entre familias (para una comparación, véase: Gutiérrez de Pineda, 1962). Usar el concepto de honor para describir un conjunto de valores de la comunidad sería erróneo si previamente no se analizan las diferencias entre las etnografías mediterráneas y la comunidad de Nómeque. Moore (1988), haciendo referencia al concepto de honor en el mundo árabe musulmán, dice: "El honor familiar depende del modo más crítico de la modesta, casta y discreta conducta sexual de hijas, hermanas y esposas" (pp. 106-107). Consideraba que este no era de ningún modo el caso de la comunidad de Nómeque; sin embargo, coincidiendo con los casos mediterráneos, nos encontrábamos con una sociedad en la que existía un fuerte despliegue y performance de un ideal de masculinidad. El choque entre dos performances masculinas compitiendo a menudo culmina en violencia. 
El concepto dominante de patronazgo existía en contraste con una fragmentada y desorganizada "alianza" de valores y prácticas que si bien no constituía un sistema completo, sí se oponía al sistema hegemónico. Llamé "madrazgo" a estas ideas y prácticas dispersas de resistencia La noción de madrazgo hacía referencia a un conjunto de valores de resistencia y a un tipo de organización familiar de los hogares que vivían en la pobreza y que estaban basados en la matrifocalidad.

Tampoco hice uso del término "matriarquía", por el abuso que se había hecho de este. Como muchos autores ya han remarcado, este abuso comenzó probablemente con la interpretación que Engels hizo de los textos de Morgan (Kuper, 1988). Por otra parte, el término "matriarquía" hace referencia a una sociedad dominada por las mujeres. "Madrazgo", en cambio, podría ser sintetizado muy esquemáticamente como una alianza entre hombres frustrados y mujeres que se quedaron a cargo de sus hogares. Las familias matrifocales eran bastante comunes en Nómeque. El término "matrifocalidad" es utilizado para describir situaciones en las que las mujeres desempeñan un rol dominante psicológica o económicamente en sus hogares, incluso en los casos en que los hombres residen en ellos (Chant, 1996). En la comunidad analizada, el rol dominante femenino contrastaba con una masculinidad agresiva expresada por los hombres que abandonaban la casa y vivían afuera. Los valores que en el madrazgo estaban adheridos a la matrifocalidad acentuaban la preocupación de las madres por sus hijos y por la administración del hogar. La matrifocalidad se encontraba en la base de la comunidad, especialmente entre sus miembros más pobres.

En mi tesis señalaba la presencia de valores de resistencia y solidaridad en la lucha campesina por la tierra. Me preocupaba el proceso de eliminación del sistema de haciendas y la división de la tierra en lotes individuales. ¿Cómo es que valores de igualdad y solidaridad fueron aceptados por hombres agresivos e individualistas? Los grupos matrifocales se encontraban sobre todo en la base de la sociedad; los valores de madrazgo estaban presentes en toda la comunidad. Discutí, además, diferentes enfoques teóricos 
de la matrifocalidad, particularmente en su relación con la pobreza y con el inestable y conflictivo carácter que frecuentemente es atribuido a este tipo de estructura familiar (Lewis, 1970, 1977; Valentine, 1968; Barros, 1994).

\section{Violencia de género}

Moore (1994) dice:

Los discursos sobre sexualidad y género construyen a las mujeres y a los hombres como a diferentes tipos de personas [...] El hecho interesante acerca de estas construcciones es que solo tienen una muy tangencial relación con las conductas, cualidades, atributos e imágenes de sí mismos de mujeres y hombres individuales (p. 138).

La masculinidad es un proceso complejo de construcción personal en relación con el otro, lo que significa al mismo tiempo confrontar representaciones culturales, no siempre homogéneas, de lo que un hombre debe ser (Wade, 1994). Si bien no siempre es claramente percibido de esta manera por los actores que nos ocupan, en Nómeque la violencia y la agresión eran algunos de los elementos más relevantes del complejo proceso de construcción de masculinidad. Así, pude observar un conflicto constante en la unidad familiar: los maridos se veían envueltos en una agresiva disputa por el poder contra sus esposas, suegras e incluso contra sus propios hijos.

Permítanme ilustrarlo con un ejemplo: la familia de Karina era un caso de familia matrifocal en conflicto y con padres ausentes. Karina era una chica de catorce años, la mayor de tres hijos. Su padre dejó la casa luego de una discusión en la que le pegó duramente a su mujer. Karina me comentó: "Mi padre solía pegar a mi madre. Una vez traté de separarlos y mi padre me tiró por las escaleras. Desde entonces tengo un pie lastimado y no camino bien". El hermano más grande defendió a su madre y peleó físicamente con su padre. Los hijos mayores a menudo se oponen a sus padres, apoyando a sus madres y compitiendo así por el rol masculino en el hogar. 
Nola Reinhardt describe estas relaciones conflictivas entre el hijo mayor y su padre o padrastro, señalando que este conflicto también explica una de las motivaciones de la migración hacia las ciudades.

En el caso de Karina, luego del incidente mencionado los chicos cuidaron de su madre y no permitieron que el padre retornara a la casa. La madre no se quería separar de él por causa de los chicos, pero finalmente se decidió a hacerlo. El padre de Karina se mudó a otro pueblo, donde comenzó una nueva relación con una mujer más joven, con la que tuvo un hijo. Más adelante también abandonó a esta mujer, para establecer una nueva relación: "Sé que ahora tiene otra china". El padre de Karina no considera como deber proveer a su familia con dinero. Argumenta que se vio obligado a irse y que entonces la culpa era de su mujer. Volvió una vez y dijo que pagaría de ser aceptado de nuevo en la casa.

\section{Violencia entre familias}

Durante mi trabajo de campo, varias venganzas de sangre tuvieron lugar en el pueblo o en sus alrededores. Las vendettas se originaban en familias -con sus propios y particulares conflictos internos- que se enfrentaban con una familia enemiga para dirimir problemas relacionados con tierras, mujeres o dinero. Fals Borda (1962) enumera las fuentes de conflicto entre campesinos que él encontró en su estudio de El Saucío: "Envidia, celos, avaricia y el no pago de deudas son motivos de conflicto y agresión con enemigos externos que también producen la cohesión de la familia” (p. 211).

Cuando una venganza comenzaba, el único interés de cada familia era la aniquilación de la otra, esto es, el asesinato de todos los varones adultos de la familia enemiga. Cada varón que quedaba vivo era una amenaza potencial para la supervivencia de la otra familia, porque siempre podía tratar de vengarse. Los asesinos pretendían "no dejar ni semilla": eliminar a esa familia de la faz de la tierra (Hobsbawm, 1982). 
Las venganzas tomaban su tiempo y los campesinos las preparaban con mucho cuidado. Como hacía notar Fals Borda (1955), "los campesinos no se vengan inmediatamente. Paciente, laboriosamente trabajan para esta [venganza]" (p. 209). Podían incluso pasar años antes de que se presentara una oportunidad para la vendetta.

\section{Narcos}

Los perfiles de los narcotraficantes estaban relacionados con los valores y prácticas que yo denominaba "patronazgo"; más aún, intenté demostrar que los narcos deseaban encarnar y actuar sobre este sistema específico de representaciones y prácticas, que aparecen ilustradas también en canciones y filmes mexicanos adoptados y adaptados por la comunidad.

¿Por qué los narcos en vez de ser condenados socialmente, encarnaban un ideal para la mayoría de los campesinos de la región? Una respuesta posible a esta pregunta estaría dada mostrando la forma en que, a través de sus actividades sociales y económicas, especialmente a través de su consumo conspicuo, los narcos reconstruían exitosamente un sistema jerárquico en una comunidad que había derrotado a los hacendados.

Los narcos de la comunidad analizada fueron campesinos pobres que obtuvieron poder y riqueza a través de la violencia. En mi tesis los definía, siguiendo a Blok (1974), como "violentos empresarios campesinos". En una comunidad inestable y no estratificada, con relaciones fluidas de poder, la violencia aparecía como un modo de establecer dominio y poder entre personas anteriormente consideradas iguales. Es interesante notar que el poder y respeto obtenido por los narcos provenía de sus propios méritos, mas no de su herencia. Es a través de la violencia como el narco construía un negocio y una red de relaciones sociales y, en definitiva, su poder. 


\section{Violencia política}

La identificación política de las familias estaba también relacionada con y condicionada por las amistades y enemistades familiares. La política y la venganza pueden estar unidas, especialmente en momentos de gran desorden político. El Estado colombiano y su sistema de seguridad y justicia quedaban fuera de estas acciones, y su intervención era sumamente limitada. En general, la gente no pedía la intervención del Estado para la resolución de sus asuntos, pues estos se decidían privadamente entre las familias en cuestión.

Por su parte, la guerrilla expresaba valores de solidaridad campesina frente a la amenaza del mundo externo, protegiendo a los más pobres tanto del Estado colombiano como de las fuerzas económicas que amenazaban su existencia.

En la comunidad de Nómeque, el Estado-nación debía luchar por el monopolio del orden social frente a otros actores sociales. No era percibido como un ente unificado, sino que la gente tenía percepciones fragmentarias de él. Existían concepciones independientes y específicas de la policía, el ejército y el sistema educativo, todas vistas como instituciones diversas y separadas. El Estado y sus instituciones tenían entonces una identidad transitoria, fragmentaria y no totalizadora en el discurso de la comunidad, lo que permitía competir a otras legalidades y a otros discursos (Pitt-Rivers, 1954).

\section{Conclusiones}

Para la comprensión de la violencia política se hace necesario pesquisar sobre la estructura social y las prácticas y representaciones culturales de la comunidad analizada. Si hablamos de violencia no podemos circunscribirla al campo específico y analíticamente separado de la política. La violencia se articula en discursos y prácticas performativas de masculinidad, jerarquía y poder. 
Las tensiones existentes dentro de la familia nos mostraban una lucha por el control del lugar. Las madres, especialmente en los estratos poblacionales más pobres, se situaban en el centro de la vida familiar (Whiteford, 1976). Una mujer exitosa conseguía ejercer un control efectivo sobre su marido, sus hijas e hijos y sus parientes políticos. En las familias más pudientes o en aquellas que poseían mayor educación formal, la participación paterna era más frecuente. Coincidiendo en este caso con lo que sugieren otras etnografías, la matrifocalidad aparecía relacionada con pobreza y con violencia doméstica (Stolcke, 1981; Lewis, 1977; Valentine, 1968).

Beber alcohol con los amigos y vivir fuera de la casa eran elementos importantes en la vida de los hombres; era también una forma de crear lazos de amistad y solidaridad entre ellos. El consumo de alcohol estaba directamente ligado a la violencia doméstica y a la agresión (Harvey y Grow, 1994; Babb, 1989). Al mismo tiempo, agresión y competición eran dos elementos centrales en la construcción social de la masculinidad. Fuertes tensiones entre los sexos son percibidas como consecuencia del contraste entre los valores de madrazgo y patronazgo. Las relaciones entre madres y yernos tienden a ser muy tensas, en una sorda lucha por el dominio del hogar, disputado en algunos casos con abierta agresión (Gutiérrez de Pineda, 1968).

En Nómeque existían tensiones dentro de la familia, por lo cual estas conclusiones intentan registrar la conexión entre los conflictos internos y la violencia externa que impregnan a la comunidad. Se trataría de una violencia permanente con un ciclo que se reproduce.

Las venganzas de sangre expresaban profundos conflictos entre y dentro de las familias campesinas, y se originaban a partir de disputas sobre los límites de las propiedades, el dinero o las mujeres (Fals Borda, 1955). Por otro lado, las vendettas eran la consecuencia de la distribución arbitraria de la tierra luego de la rebelión agraria y mostraban que aún no se había constituido una nueva jerarquía establecida. La división de la tierra en lotes individuales y la falta de una organización central implicaron el uso de la violencia 
como forma de definición de los conflictos entre familias. Por otro lado, las vendettas significaban un intento de constituir nuevas jerarquías: surgían en un espacio igualitario, pero buscaban imponer la autoridad y la superioridad de una de las facciones envueltas. Las vendettas eran la consecuencia de la violencia y agresión masculinas en el proceso de intentar ser "verdaderos hombres"; podríamos decir que eran intentos frustrados de ser patrones de hacienda. Incluso después de la rebelión agraria, los campesinos pobres continuaron compitiendo entre sí y sus valores de solidaridad e igualdad no sirvieron para terminar con la violencia.

Además, las vendettas reforzaban la solidaridad entre parientes: las relaciones hacia adentro de la familia se fortalecían y las relaciones hacia fuera de ella se debilitaban. Cuando el miembro de una familia era asesinado, esta trataba de matar todo miembro masculino posible de la otra. Al matar a los miembros masculinos, se reducían las posibilidades de futuras venganzas (Hobsbawm, 1983, p. 270). En la mayoría de los casos, la familia extendida era el grupo mínimo que expresaba solidaridad y participaba activamente en la venganza, a causa de una acción cometida contra uno de sus miembros (Black-Michaud, 1975; Fals Borda, 1955). Sin embargo, en algunos casos particulares ni siquiera la solidaridad familiar podía detener la competencia entre hombres, y esta podía producir divisiones en el seno de la misma familia.

Las familias envueltas en vendettas solían tener problemas con sus vecinos y establecían una relación de odio con sus enemigos (Reichel-Dolmatoff, 1961). Estos grupos, que estaban generalmente compuestos por un importante número de hermanos varones o primos hermanos varones, eran temidos por la comunidad, ya que presentaban una estructura interna de marcada conflictividad. A través de la venganza, estos problemas internos pasaban a segundo plano. Eran los hogares con conflictos intestinos los que participaban de la violencia entre linajes; así, las relaciones con otras familias en muchos casos se trasladaban a la disputa política, conectándose de este modo lo interno con lo externo. Las vendettas eran la llave para entender 
la relación entre la comunidad y las fuerzas externas. Existía un in crescendo desde las venganzas familiares a la competencia política, y la ideología familiar era transformada en ideología política. Lo central es entender que si hablamos de violencia no podemos circunscribirla al ámbito específico y analíticamente separado de la política.

Este ejemplo en particular nos hace ver, por un lado, que la autonomía de la política es relativa y, por otro lado, que la resolución de un problema en la esfera política puede ser una resolución parcial. De hecho, muchos acuerdos de paz, como por ejemplo el de El Salvador, que elogiaba Bejarano, resolvieron la violencia política, pero no impidieron la generalización de una violencia delictiva con niveles altísimos de homicidios. Esto nos remite a la pregunta sobre cómo crear canales que conecten la investigación y la práctica, sin sentirnos abrumados por la complejidad de un problema que se presenta como una hidra con mil cabezas. No tengo una respuesta terminante, pero considero que se tiene que intentar comprender la existencia de elementos articulados subyacentes al problema central, al igual que preparar una respuesta que los contemple. En el caso que hemos desarrollado con mayor detalle, la violencia se articula en discursos de masculinidad, jerarquía y poder, y es solo una comprensión de tales relaciones y clivajes lo que nos permitirá comunicar un discurso que busque construir una paz duradera.

\section{Referencias}

Álvarez, S. (2002). La distancia en el discurso profesional de la justicia argentina: la representación de la criminalidad en la justicia penal ante la "nueva ola" de violencia delictiva. En Gayol, S. y Kessler, G. (comps.). Violencias, delitos y justicias en la Argentina. Buenos Aires: Manantial - Universidad de General Sarmiento.

Babb, F. E. (1989). Between field and cooking pot: the political economy of market women in Perú. Austin: Texas University Press. 
Barros, R., Fox, L. \& Mendonca, R. (1994). Female headed households. Poverty, and the welfare of children in urban Brazil. Washington: The World Bank Policy Research Department.

Black-Michaud, J. (1975). Feuding societies. Oxford: Basil Blackwell.

Block, A. (1975). The mafia of a Sicilian village, 1860-1960. A study of violent peasant entrepreneurs. London/New York: Harper and Row.

Bonaldi, P. (2002). Evolución de las muertes violentas en la Argentina, 1980-1999. En Gayol, S. y Kessler, G. (comps.). Violencias, delitos y justicias en la Argentina. Buenos Aires: Manantial - Universidad de General Sarmiento.

Chant, S. (1996). Women-headed households, diversity and dynamics in the developing world. Londres: Macmillan Press.

Cruz Artacho, S. (1994). Caciques y campesinos. Poder político, modernización agraria y conflictividad rural en Granada, 1890-1923. Córdoba: Ediciones Libertarias.

Evans-Pritchard, E. E. (1977). Los Nuer. Barcelona: Anagrama.

Fals Borda, O. (1962). Peasant society in the Colombian Andes: a sociological study of Saucio. Gainesville: University of Florida Press.

Gutiérrez de Pineda, V. (1962). La familia en Colombia. Estudio antropológico. Bogotá: Estudios Sociológicos Latinoamericanos.

Gutiérrez de Pineda, V. (1975). Familia y cultura en Colombia. Bogotá: Colcultura.

Gutiérrez de Pineda, V. (1976). Estructura, función y cambio de la familia en Colombia. Bogotá: Asociación Colombiana de Facultades de Medicina.

Harvey, P. \& Gow, P. (1994). Introduction. En Sex and violence. Issues in representation and experience. Londres: Routledge. 
Hobsbawm, E. (1982). La anatomía de la violencia en Colombia. En Rebeldes primitivos. Barcelona: Ariel.

Kuper, A. (1988). The invention of primitive society. Transformations of an illusion. London: Routledge

Lewis, O. (1961). The children of Sánchez. New York: Random House.

Lewis, O. (1977). Antropología de la pobreza. México: Fondo de Cultura Económica.

Marvin, G. (1988). Honor, integridad y el problema de la violencia en la corrida de toros en España. En Riches, D. (Ed.). El fenómeno de la violencia. Madrid: Pirámide.

Melhuus, M. y Stølen, K. A. (2008). Machos, putas, santas. El poder del imaginario en América Latina. Buenos Aires: Antropofagia.

Mintz, S. \& Wolf, E. (1957). Haciendas and plantations in Middle America and the Antilles. Social and Economic Studies, 6, 380-412.

Moore, H. (1994). The problem of explaining violence. En Harvey, P. \& Gow, P. (Ed.). Sex and violence. Issues in representation and experience. Nueva York: Routledge.

Moore, H. (1988). Feminism and anthropology. Cambridge: Polity Press.

Peristiany, J. G. \& Pitt-Rivers, J. (1992). Honor and grace in anthropology. Cambridge: Cambridge University Press.

Pitt Rivers, J. (1954). The People of the Sierra. Chicago: University of Chicago Press.

Reichel Dolmatoff, G. \& Reichel Dolmatoff, A. (1961). People of Aritama. The cultural personality of a Colombian mestizo village. Chicago: The University of Chicago Press. 
Reinhart, N. (1988). Our daily bread. Berkeley University of California Press.

Riches, D. (1988). El fenómeno de la violencia. Madrid: Pirámide.

Stevens, E. P. (1973). Marianismo: the other face of machismo in Latin America. En Pescatello, A. (Ed.). Female and male in Latin America. Londres: University of Pittsburgh Press.

Stolcke, V. (1981). Women's labours: the naturalisation of social inequality and women's subordination. En Young, K., Wolkowitz, G. \& Mccullagh, R. (Ed.). Of marriage and the market: women's subordination in international perspective. London: CSE Books.

Stølen, K. A. (1991). Gender, sexuality and violence in Ecuador. En Gender, culture, and power in developing countries. Oslo: Centre for Development and Environment.

Valentine, C. A. (1968). Culture and poverty. Chicago: University of Chicago Press.

Wade, P. (1994). Man the hunter, gender and violence in music and drinking contexts in Colombia. En Sex and violence. Issues in representation and experience. Londres: Routledge.

Whiteford, M. (1976). The forgotten ones. Colombian countrymen in an urban setting. Gainesville: The University Press of Florida. 
